\title{
Research Article \\ On a Hyperbolic Coefficient Inverse Problem via Partial Dynamic Boundary Measurements
}

\author{
Christian Daveau, ${ }^{1}$ Diane Manuel Douady, ${ }^{1}$ \\ and Abdessatar Khelifi ${ }^{2}$ \\ ${ }^{1}$ Département de Mathématiques, CNRS AGM UMR 8088, Université de Cergy-Pontoise, \\ 95302 Cergy-Pontoise Cedex, France \\ 2 Département de Mathématiques, Université des Sciences de Carthage, 7021 Bizerte, Tunisia \\ Correspondence should be addressed to Christian Daveau, christian.daveau@u-cergy.fr
}

Received 29 March 2010; Revised 31 May 2010; Accepted 1 June 2010

Academic Editor: Christo I. Christov

Copyright (C) 2010 Christian Daveau et al. This is an open access article distributed under the Creative Commons Attribution License, which permits unrestricted use, distribution, and reproduction in any medium, provided the original work is properly cited.

This paper is devoted to the identification of the unknown smooth coefficient $c$ entering the hyperbolic equation $c(x) \partial_{t}^{2} u-\Delta u=0$ in a bounded smooth domain in $\mathbb{R}^{d}$ from partial (on part of the boundary) dynamic boundary measurements. In this paper, we prove that the knowledge of the partial Cauchy data for this class of hyperbolic PDE on any open subset $\Gamma$ of the boundary determines explicitly the coefficient $c$ provided that $c$ is known outside a bounded domain. Then, through construction of appropriate test functions by a geometrical control method, we derive a formula for calculating the coefficient $c$ from the knowledge of the difference between the local Dirichlet-to-Neumann maps.

\section{Introduction}

In this paper, we present a new method for multidimensional Coefficient Inverse Problems (CIPs) for a class of hyperbolic Partial Differential Equations (PDEs). In the literature, the reader can find many key investigations of this kind of inverse problems; see, for example, [1-11] and references cited there. Beilina and Klibanov have deeply studied this important problem in various recent works [2, 12]. In [2], the authors have introduced a new globally convergent numerical method to solve a coefficient inverse problem associated to a hyperbolic PDE. The development of globally convergent numerical methods for multidimensional CIPs has started, as a first generation, from the developments found in [13-15]. Else, Ramm and Rakesh have developed a general method for proving uniqueness theorems for multidimensional inverse problems. For 
the two dimensional case, Nachman [7] proved a uniqueness result for CIPs for some elliptic equation. Moreover, we find the works of Päivärinta and Serov [16, 17] about the same issue, but for elliptic equations. In other manner, the author Chen has treated in [18] the Fourier transform of the hyperbolic equation similar to ours with the unknown coefficient $c(x)$. Unlike this, we derive, using as weights particular background solutions constructed by a geometrical control method, asymptotic formulas in terms of the partial dynamic boundary measurements (Dirichlet-to-Neumann map) that are caused by the small perturbations. These asymptotic formulae yield the inverse Fourier transform of unknown coefficient.

The ultimate objective of the work described in this paper is to determine, effectively, the unknown smooth coefficient $c$ entering a class of hyperbolic equations in a bounded smooth domain in $\mathbb{R}^{d}$ from partial (on part of the boundary) dynamic boundary measurements. The main difficulty which appears in boundary measurements is that the formulation of our boundary value problem involves unknown boundary values. This problem is well known in the study of the classical elliptic equations, where the characterization of the unknown Neumann boundary value in terms of the given Dirichlet datum is known as the Dirichlet-to-Neumann map. But, the problem of determining the unknown boundary values also occurs in the study of hyperbolic equations formulated in a bounded domain.

As our main result, we develop, using as weights particular background solutions constructed by a geometrical control method, asymptotic formulas for appropriate averaging of the partial dynamic boundary measurements that are caused by the small perturbations of coefficient according to a parameter $\alpha$.

The final formula (3.44) represents a promising approach to the dynamical identification and reconstruction of the coefficient $c(x)$. Moreover, it improves the given asymptotic formula (2.3) of the coefficient $c(x)$. Assume that the coefficient is known outside a bounded domain $\Omega$, and suppose that we know explicitly the value of $\lim _{\alpha \rightarrow 0^{+}} c(x)$ for $x \in \Omega$. Then, the developed asymptotic formulae yield the inverse Fourier transform of the unknown part of this coefficient.

In the subject of small volume perturbations from a known background material associated to the full time-dependent Maxwell's equations, we have derived asymptotic formulas to identify their locations and certain properties of their shapes from dynamic boundary measurements [19]. The present paper represents a different investigation of this line of work.

As closely related stationary identification problems, we refer the reader to [7, 20-22] and references cited there.

\section{Problem Formulation}

Let $\Omega \subset \mathbb{R}^{d}$ be a bounded domain with a smooth boundary and let $d=2,3$ (our assumption $d \leq 3$ is necessary in order to obtain the appropriate regularity for the solution using classical Sobolev embedding; see Brezis [23]). For simplicity, we take $\partial \Omega$ to be $\mathcal{C}^{\infty}$, but this condition could be considerably weakened. Let $n=n(x)$ denote the outward unit normal vector to $\Omega$ at a point on $\partial \Omega$. Let $T>0, x_{0} \in \mathbb{R}^{d} \backslash \bar{\Omega}$, and let $\Omega^{\prime}$ be a smooth subdomain of $\Omega$. We denote by $\Gamma \subset \subset \partial \Omega$ a measurable smooth open part of the boundary $\partial \Omega$.

Throughout this paper, we will use quite standard $L^{2}$-based Sobolev spaces to measure regularity. 
As the forward problem, we consider the initial boundary value problem for a hyperbolic PDE in the domain $\Omega \times(0, T)$

$$
\begin{gathered}
\left(c_{\alpha} \partial_{t}^{2}-\Delta\right) u_{\alpha}=0, \quad \text { in } \Omega \times(0, T), \\
\left.u_{\alpha}\right|_{t=0}=\varphi,\left.\quad \partial_{t} u_{\alpha}\right|_{t=0}=\psi \quad \text { in } \Omega, \\
\left.u_{\alpha}\right|_{\partial \Omega \times(0, T)}=f .
\end{gathered}
$$

Here $\varphi, \psi \in \mathcal{C}^{\infty}(\bar{\Omega})$ and $f \in \mathcal{C}^{\infty}\left(0, T ; \mathcal{C}^{\infty}(\partial \Omega)\right)$ are subject to the compatibility conditions

$$
\left.\partial_{t}^{2 l} f\right|_{t=0}=\left.\left.\left(\Delta^{l} \varphi\right)\right|_{\partial \Omega^{\prime}} \quad \partial_{t}^{2 l+1} f\right|_{t=0}=\left.\left(\Delta^{l} \psi\right)\right|_{\partial \Omega^{\prime}} \quad l=1,2, \ldots
$$

which give that (2.6) has a unique solution in $\mathcal{C}^{\infty}([0, T] \times \bar{\Omega})$; see [24]. It is also well known that (2.1) has a unique weak solution $u_{\alpha} \in \mathcal{C}^{0}\left(0, T ; H^{1}(\Omega)\right) \cap \mathcal{C}^{1}\left(0, T ; L^{2}(\Omega)\right)$; see [24, 25]. Indeed, from [25] we have that $\left.\left(\partial u_{\alpha} / \partial n\right)\right|_{\partial \Omega}$ belongs to $L^{2}\left(0, T ; L^{2}(\partial \Omega)\right)$.

Equation (2.1) governs a wide range of applications, including, for example, propagation of acoustic and electromagnetic waves.

We assume that the coefficient $c(x)$ of $(2.1)$ is such that

$$
c(x)= \begin{cases}c_{\alpha}(x)=c_{0}(x)+\alpha c_{1}(x), & \text { for } x \in \Omega, \\ c_{2}(x)=\text { const. }>0, & \text { for } x \in \mathbb{R}^{d} \backslash \Omega,\end{cases}
$$

where $c_{i}(x) \in \mathcal{C}^{2}(\bar{\Omega})$ for $i=0,1$ with

$$
c_{1} \equiv 0 \quad \text { in } \Omega \backslash \bar{\Omega}^{\prime}, \quad M:=\sup \left\{c_{1}(x) ; x \in \Omega^{\prime}\right\},
$$

where $\Omega^{\prime}$ is a smooth subdomain of $\Omega$ and $M$ is a positive constant. We also assume that $\alpha>0$, the order of magnitude of the small perturbations of coefficient, is sufficiently small that

$$
\left|c_{\alpha}(x)\right| \geq c_{*}>0, \quad x \in \bar{\Omega},
$$

where $c_{*}$ is a positive constant.

Define $u$ to be the solution of the hyperbolic equation in the homogeneous situation $(\alpha=0)$. Thus, $u$ satisfies

$$
\begin{gathered}
\left(c_{0} \partial_{t}^{2}-\Delta\right) u=0, \quad \text { in } \Omega \times(0, T), \\
\left.u\right|_{t=0}=\varphi,\left.\quad \partial_{t} u\right|_{t=0}=\psi \quad \text { in } \Omega, \\
\left.u\right|_{\partial \Omega \times(0, T)}=f .
\end{gathered}
$$


Now, we define $\Gamma_{c}:=\partial \Omega \backslash \bar{\Gamma}$, and we introduce the trace space

$$
\widetilde{H}^{1 / 2}(\Gamma)=\left\{v \in H^{1 / 2}(\partial \Omega \times(0, T)), v \equiv 0 \text { on } \Gamma_{c} \times(0, T)\right\} .
$$

It is well known that the dual of $\widetilde{H}^{1 / 2}(\Gamma)$ is $H^{-1 / 2}(\Gamma)$.

Then, one can write

$$
\Lambda_{\alpha}\left(\left.f\right|_{\Gamma}\right)=\left.\frac{\partial u_{\alpha}}{\partial n}\right|_{\Gamma}, \quad \text { for }\left.f\right|_{\Gamma} \in \widetilde{H}^{1 / 2}(\Gamma)
$$

where $\Lambda_{\alpha}$ is the Dirichlet-to-Neumann map (D-t-N) operator, and $u_{\alpha}$ is the solution of (2.1). Let $\Lambda_{0}$ be the Dirichlet-to-Neumann map (D-t-N) operator defined as in (2.8) for the case $\alpha=0$. Then, our problem can be stated as follows.

\section{Inverse Problem}

Suppose that the smooth coefficient $c(x)$ satisfies (2.4), (2.5), and (2.6), where the positive number $c_{2}$ is given. Assume that the function $c(x)$ is unknown in the domain $\Omega$. Is it possible to determine the coefficient $c_{\alpha}(x)$ from the knowledge of the difference between the local Dirichlet-to-Neumann maps $\Lambda_{\alpha}-\Lambda_{0}$ on $\Gamma$, if we know explicitly the value of $\lim _{\alpha \rightarrow 0^{+}} \mathcal{C}_{\alpha}(x)$ for $x \in \Omega$ ?

To give a positive answer, we will develop an asymptotic expansions of an "appropriate averaging" of $\partial u_{\alpha} / \partial n$ on $\Gamma \times(0, T)$, using particular background solutions as weights. These particular solutions are constructed by a control method as it has been done in the original work [10] (see also [11, 26-29]). It has been known for some time that the full knowledge of the (hyperbolic) Dirichlet to Neumann map $\left(\left.\left.u_{\alpha}\right|_{\partial \Omega \times(0, T)} \mapsto\left(\partial u_{\alpha} / \partial n\right)\right|_{\partial \Omega \times(0, T)}\right)$ uniquely determines conductivity; see [30,31]. Our identification procedure can be regarded as an important attempt to generalize the results of $[30,31]$ in the case of partial knowledge (i.e., on only part of the boundary) of the Dirichlet-to-Neumann map to determine the coefficient of the hyperbolic equation considered above. The question of uniqueness of this inverse problem can be addressed positively via the method of Carleman estimates; see, for example, $[6,14]$.

\section{The Identification Procedure}

Before describing our identification procedure, let us introduce the following cutoff function $\beta(x) \in \mathcal{C}_{0}^{\infty}(\Omega)$ such that $\beta \equiv 1$ on $\Omega^{\prime}$ and let $\eta \in \mathbb{R}^{d}$.

We will take in what follows $\varphi(x)=e^{i \eta \cdot x}, \psi(x)=-i|\eta| e^{i \eta \cdot x}$, and $f(x, t)=e^{i \eta \cdot x-i|\eta| t}$ and assume that we are in possession of the boundary measurements of

$$
\frac{\partial u_{\alpha}}{\partial n} \quad \text { on } \Gamma \times(0, T)
$$

This particular choice of data $\varphi, \psi$, and $f$ implies that the background solution $u$ of the wave equation (2.6) in the homogeneous background medium can be given explicitly. 
Suppose now that $T$ and the part $\Gamma$ of the boundary $\partial \Omega$ are such that they geometrically control $\Omega$ which roughly means that every geometrical optic ray, starting at any point $x \in \Omega$ at time $t=0$, hits $\Gamma$ before time $T$ at a nondiffractive point; see [32]. It follows from [33] (see also [34]) that there exists (a unique) $g_{\eta} \in H_{0}^{1}\left(0, T ; T L^{2}(\Gamma)\right.$ ) (constructed by the Hilbert Uniqueness Method) such that the unique weak solution $w_{\eta}$ to the wave equation

$$
\begin{gathered}
\left(c_{0} \partial_{t}^{2}-\Delta\right) w_{\eta}=0 \quad \text { in } \Omega \times(0, T), \\
\left.w_{\eta}\right|_{t=0}=\beta(x) e^{i \eta \cdot x} \in H_{0}^{1}(\Omega), \\
\left.\partial_{t} w_{\eta}\right|_{t=0}=0 \quad \text { in } \Omega, \\
\left.w_{\eta}\right|_{\Gamma \times(0, T)}=g_{\eta^{\prime}} \\
\left.w_{\eta}\right|_{\partial \Omega \mid \bar{\Gamma} \times(0, T)}=0
\end{gathered}
$$

satisfies $w_{\eta}(T)=\partial_{t} w_{\eta}(T)=0$.

Let $\theta_{\eta} \in H^{1}\left(0, T ; L^{2}(\Gamma)\right)$ denote the unique solution of the Volterra equation of second kind

$$
\begin{gathered}
\partial_{t} \theta_{\eta}(x, t)+\int_{t}^{T} e^{-i|\eta|(s-t)}\left(\theta_{\eta}(x, s)-i|\eta| \partial_{t} \theta_{\eta}(x, s)\right) d s=g_{\eta}(x, t), \quad \text { for } x \in \Gamma, t \in(0, T), \\
\theta_{\eta}(x, 0)=0, \quad \text { for } x \in \Gamma .
\end{gathered}
$$

We can refer to the work of Yamamoto in [11] who conceived the idea of using such Volterra equation to apply the geometrical control for solving inverse source problems.

The existence and uniqueness of this $\theta_{\eta}$ in $H^{1}\left(0, T ; L^{2}(\Gamma)\right)$ for any $\eta \in \mathbb{R}^{d}$ can be established using the resolvent kernel. However, observing from differentiation of (3.3) with respect to $t$ that $\theta_{\eta}$ is the unique solution of the ODE:

$$
\begin{gathered}
\partial_{t}^{2} \theta_{\eta}-\theta_{\eta}=e^{i|\eta| t} \partial_{t}\left(e^{-i|\eta| t} g_{\eta}\right), \quad \text { for } x \in \Gamma, t \in(0, T), \\
\theta_{\eta}(x, 0)=0, \quad \partial_{t} \theta_{\eta}(x, T)=0, \quad \text { for } x \in \Gamma
\end{gathered}
$$

the function $\theta_{\eta}$ may be found (in practice) explicitly with variation of parameters and it also immediately follows from this observation that $\theta_{\eta}$ belongs to $H^{2}\left(0, T ; L^{2}(\Gamma)\right)$.

We introduce $v_{\eta}$ as the unique weak solution (obtained by transposition) in $\mathcal{C}^{0}\left(0, T ; L^{2}(\Omega)\right) \cap \mathcal{C}^{1}\left(0, T ; H^{-1}(\Omega)\right)$ to the wave equation

$$
\begin{gathered}
\left(c_{0} \partial_{t}^{2}-\Delta\right) v_{\eta}=0 \quad \text { in } \Omega \times(0, T), \\
\left.v_{\eta}\right|_{t=0}=0 \quad \text { in } \Omega, \\
\left.\partial_{t} v_{\alpha, \eta}\right|_{t=0}=i \nabla \cdot\left(\eta c_{1}(x) e^{i \eta \cdot x}\right) \in L^{2}(\Omega), \\
\left.v_{\eta}\right|_{\partial \Omega \times(0, T)}=0 .
\end{gathered}
$$

Then, the following holds. 
Proposition 3.1. Suppose that $\Gamma$ and $T$ geometrically control $\Omega$. For any $\eta \in \mathbb{R}^{d}$, we have

$$
\int_{0}^{T} \int_{\Gamma} g_{\eta} \Lambda_{0}\left(v_{\eta}\right) d \sigma(x) d t=|\eta|^{2} \int_{\Omega^{\prime}} c_{1}(x) e^{2 i \eta \cdot x} d x
$$

Here $d \sigma(x)$ means an elementary surface for $x \in \Gamma$.

Proof. Let $v_{\eta}$ be the solution of (3.5). From [25, Theorem 4.1, page 44], it follows that $\Lambda_{0}\left(v_{\eta}\right)=\left.\left(\partial v_{\eta} / \partial n\right)\right|_{\Gamma} \in L^{2}\left(0, T ; L^{2}(\Gamma)\right)$. Then, multiplying the equation $\left(\partial_{t}^{2}+\Delta\right) v_{\eta}=0$ by $w_{\eta}$ and integrating by parts over $(0, T) \times \Omega$, for any $\eta \in \mathbb{R}^{d}$, we have

$$
\int_{0}^{T} \int_{\Omega}\left(\partial_{t}^{2}-\Delta\right) v_{\eta} w_{\eta}=i \int_{\Omega} \nabla \cdot\left(\eta c_{1}(x) e^{i \eta \cdot x}\right) \beta(x) e^{i \eta \cdot x} d x-\int_{0}^{T} \int_{\Gamma} g_{\eta} \frac{\partial v_{\eta}}{\partial n}=0 .
$$

Therefore,

$$
|\eta|^{2} \int_{\Omega^{\prime}} c_{1}(x) e^{2 i \eta \cdot x} d x=\int_{0}^{T} \int_{\Gamma} g_{\eta} \frac{\partial v_{\eta}}{\partial n}
$$

since $c_{1} \equiv 0$ on $\Omega \backslash \overline{\Omega^{\prime}}$.

In terms of the function $v_{\eta}$ as solution of (3.3), we introduce

$$
\tilde{u}_{\alpha}(x, t)=u(x, t)+\alpha^{d} \int_{0}^{t} e^{-i|\eta| s} v_{\eta}(x, t-s) d s, \quad x \in \Omega, t \in(0, T) .
$$

Moreover, for $z(t) \in \mathcal{C}_{0}^{\infty}(] 0, T[)$ and for any $v \in L^{1}\left(0, T ; L^{2}(\Omega)\right)$, we define

$$
\widehat{v}(x)=\int_{0}^{T} v(x, t) z(t) d t \in L^{2}(\Omega) .
$$

The following lemma is useful to prove our main result.

Lemma 3.2. Consider an arbitrary function $c(x)$ satisfying condition (2.3), and assume that conditions (2.4) and (2.5) hold. Let $u$ and $u_{\alpha}$ be solutions of (2.6) and (2.1), respectively. Then, using (3.9) the following estimates hold:

$$
\left\|u_{\alpha}-u\right\|_{L^{\infty}\left(0, T ; L^{2}(\Omega)\right)} \leq C \alpha
$$

where $C$ is a positive constant. And

$$
\left\|\tilde{u}_{\alpha}-u_{\alpha}\right\|_{L^{\infty}\left(0, T ; L^{2}(\Omega)\right)} \leq C^{\prime} \alpha^{d+1}
$$

where $C^{\prime}$ is a positive constant. 
Proof. Let $y_{\alpha}$ be defined by

$$
\begin{gathered}
y_{\alpha} \in H_{0}^{1}(\Omega), \\
\Delta y_{\alpha}=c_{\alpha} \partial_{t}\left(u_{\alpha}-u\right) \quad \text { in } \Omega .
\end{gathered}
$$

We have

$$
\int_{\Omega} c_{\alpha} \partial_{t}^{2}\left(u_{\alpha}-u\right) y_{\alpha}+\int_{\Omega} \nabla\left(u_{\alpha}-u\right) \cdot \nabla y_{\alpha}=\alpha \int_{\Omega} \frac{c_{1}}{c_{0}} \nabla u \cdot \nabla y_{\alpha}
$$

Since

$$
\begin{gathered}
\int_{\Omega} \nabla\left(u_{\alpha}-u\right) \cdot \nabla y_{\alpha}=-\int_{\Omega} c_{\alpha} \partial_{t}\left(u_{\alpha}-u\right)\left(u_{\alpha}-u\right)=-\frac{1}{2} \partial_{t} \int_{\Omega} c_{\alpha}\left(u_{\alpha}-u\right)^{2}, \\
\int_{\Omega} c_{\alpha} \partial_{t}^{2}\left(u_{\alpha}-u\right) y_{\alpha}=-\frac{1}{2} \partial_{t} \int_{\Omega}\left|\nabla y_{\alpha}\right|^{2},
\end{gathered}
$$

we obtain

$$
\partial_{t} \int_{\Omega}\left|\nabla y_{\alpha}\right|^{2}+\partial_{t} \int_{\Omega} c_{\alpha}\left(u_{\alpha}-u\right)^{2}=-2 \alpha \int_{\Omega} \frac{c_{1}}{c_{0}} \nabla u \cdot \nabla y_{\alpha} \leq C \alpha\left\|\nabla y_{\alpha}\right\|_{L^{\infty}\left(0, T ; L^{2}(\Omega)\right)}
$$

From the Gronwall Lemma, it follows that

$$
\left\|u_{\alpha}-u\right\|_{L^{\infty}\left(0, T ; L^{2}(\Omega)\right)} \leq C \alpha
$$

As a consequence, by using (3.10), one can see that the function $\widehat{u}_{\alpha}-\widehat{u}$ solves the following boundary value problem:

$$
\begin{gathered}
\Delta\left(\widehat{u}_{\alpha}-\widehat{u}\right)=O(\alpha) \quad \text { in } \Omega, \\
\left.\left(\widehat{u}_{\alpha}-\widehat{u}\right)\right|_{\partial \Omega}=0 .
\end{gathered}
$$

Integration by parts immediately gives

$$
\left\|\operatorname{grad}\left(\widehat{u}_{\alpha}-\widehat{u}\right)\right\|_{L^{2}(\Omega)}=O(\alpha)
$$

Taking into account that $\operatorname{grad}\left(u_{\alpha}-u\right) \in L^{\infty}\left(0, T ; L^{2}(\Omega)\right)$, we find by using the above estimate that

$$
\left\|\operatorname{grad}\left(u_{\alpha}-u\right)\right\|_{L^{2}(\Omega)}=O(\alpha) \quad \text { a.e. } t \in(0, T)
$$


Under relation (3.9), one can define the function $\tilde{y}_{\alpha}$ as a solution of

$$
\begin{gathered}
\tilde{y}_{\alpha} \in H_{0}^{1}(\Omega), \\
\Delta \tilde{y}_{\alpha}=c_{\alpha} \partial_{t}\left(\tilde{u}_{\alpha}-u_{\alpha}\right) \quad \text { in } \Omega .
\end{gathered}
$$

Integrating by parts immediately yields

$$
\begin{gathered}
\int_{\Omega} c_{\alpha} \partial_{t}^{2}\left(\tilde{u}_{\alpha}-u_{\alpha}\right) \tilde{y}_{\alpha}=-\frac{1}{2 \partial_{t}} \int_{\Omega}\left|\nabla \tilde{y}_{\alpha}\right|^{2} \\
\int_{\Omega} \nabla\left(\tilde{u}_{\alpha}-u_{\alpha}\right) \nabla \tilde{y}_{\alpha}=-\frac{1}{2 \partial_{t}} \int_{\Omega} c_{\alpha}\left(\tilde{u}_{\alpha}-u_{\alpha}\right)^{2} .
\end{gathered}
$$

To proceed with the proof of estimate (3.12), we firstly remark that the function $\tilde{u}_{\alpha}$ given by (3.9) is a solution of

$$
\begin{gathered}
\left(c_{0} \partial_{t}^{2}-\Delta\right) \tilde{u}_{\alpha}=i \alpha^{d} \nabla \cdot\left(\eta c_{1}(x) e^{i \eta \cdot x}\right) e^{-i|\eta| t} \in L^{2}(\Omega) \text { in } \Omega \times(0, T), \\
\left.\tilde{u}_{\alpha}\right|_{t=0}=\varphi(x) \text { in } \Omega, \\
\left.\partial_{t} \tilde{u}_{\alpha}\right|_{t=0}=\psi(x) \quad \text { in } \Omega, \\
\left.\tilde{u}_{\alpha}\right|_{\partial \Omega \times(0, T)}=e^{i \eta \cdot x-i|\eta| t}
\end{gathered}
$$

Then, we deduce that $u_{\alpha}-\tilde{u}_{\alpha}$ solves the following initial boundary value problem:

$$
\begin{gathered}
\left(c_{\alpha} \partial_{t}^{2}-\nabla \cdot \Delta\right)\left(u_{\alpha}-\tilde{u}_{\alpha}\right)=\alpha^{d} \nabla \cdot\left(c_{1}(x) \operatorname{grad}\left(\int_{0}^{t} e^{-i|\eta| s} v_{\eta}(x, t-s) d s\right)\right) \text { in } \Omega \times(0, T), \\
\left.\left(u_{\alpha}-\tilde{u}_{\alpha}\right)\right|_{t=0}=0 \text { in } \Omega, \\
\left.\partial_{t}\left(u_{\alpha}-\tilde{u}_{\alpha}\right)\right|_{t=0}=0 \text { in } \Omega, \\
\left.\left(u_{\alpha}-\tilde{u}_{\alpha}\right)\right|_{\partial \Omega \times(0, T)}=0 .
\end{gathered}
$$

Finally, we can use (3.24) to find by integrating by parts that

$$
\partial_{t} \int_{\Omega}\left|\nabla \tilde{y}_{\alpha}\right|^{2}+\partial_{t} \int_{\Omega} c_{\alpha}\left(\tilde{u}_{\alpha}-u_{\alpha}\right)^{2}=2 \alpha^{d} \int_{\Omega} c_{1} \operatorname{grad}\left(u-u_{\alpha}\right) \cdot \operatorname{grad} \tilde{y}_{\alpha}
$$

which, from the Gronwall Lemma and by using (3.20), yields

$$
\left\|\tilde{u}_{\alpha}-u_{\alpha}\right\|_{L^{\infty}\left(0, T ; L^{2}(\Omega)\right)} \leq C^{\prime} \alpha^{d+1} .
$$

This achieves the proof. 
Now, we identify the function $c(x)$ by using the difference between local Dirichlet to Neumann maps and the function $\theta_{\eta}$ as a solution to the Volterra equation (3.3) or equivalently the ODE (3.4), as a function of $\eta$. Then, the following main result holds.

Theorem 3.3. Let $\eta \in \mathbb{R}^{d}, d=2$,3. Suppose that the smooth coefficient $c(x)$ satisfies (2.3), (2.4), and (2.5). Let $u_{\alpha}$ be the unique solution in $\mathcal{C}^{0}\left(0, T ; H^{1}(\Omega)\right) \cap \mathcal{C}^{1}\left(0, T ; L^{2}(\Omega)\right)$ to the wave equation (2.1) with $\varphi(x)=e^{i \eta \cdot x}, \psi(x)=-i|\eta| e^{i \eta \cdot x}$, and $f(x, t)=e^{i \eta \cdot x-i|\eta| t}$. Let $\tilde{f}=\left.f\right|_{\Gamma} \in \widetilde{H}^{1 / 2}(\Gamma)$. Suppose that $\Gamma$ and $T$ geometrically control $\Omega$; then we have

$$
\begin{aligned}
\int_{0}^{T} \int_{\Gamma}\left(\theta_{\eta}+\partial_{t} \theta_{\eta} \partial_{t}\right)\left(\Lambda_{\alpha}-\Lambda_{0}\right)(\tilde{f})(x, t) d \sigma(x) d t & =\alpha^{d-1}|\eta|^{2} \int_{\Omega^{\prime}}\left(c_{\alpha}-c_{0}\right)(x) e^{2 i \eta \cdot x} d x+O\left(\alpha^{d+1}\right) \\
& =\alpha^{d}|\eta|^{2} \int_{\Omega^{\prime}} c_{1}(x) e^{2 i \eta \cdot x} d x+O\left(\alpha^{d+1}\right)
\end{aligned}
$$

where $\theta_{\eta}$ is the unique solution to the ODE (3.4) with $g_{\eta}$ defined as the boundary control in (3.2). The term $O\left(\alpha^{d+1}\right)$ is independent of the function $c_{1}$. It depends only on the bound $M$.

Proof. Since the extension of $\left(\Lambda_{\alpha}-\Lambda_{0}\right)(\tilde{f})(x, t)$ to $\partial \Omega \times(0, T)$ is $\left(\partial u_{\alpha} / \partial n-\partial u / \partial n\right)$, then by conditions $\partial_{t} \theta_{\eta}(T)=0$ and $\left.\left(\partial u_{\alpha} / \partial n-\partial u / \partial n\right)\right|_{t=0}=0$, we have $\left.\left(\Lambda_{\alpha}-\Lambda_{0}\right)(\tilde{f})(x, t)\right|_{t=0}=0$. Therefore, the term

$$
\int_{0}^{T} \int_{\Gamma} \partial_{t} \theta_{\eta} \partial_{t}\left(\Lambda_{\alpha}-\Lambda_{0}\right)(\tilde{f})(x, t) d \sigma(x) d t
$$

may be simplified as follows:

$$
\int_{0}^{T} \int_{\Gamma} \partial_{t} \theta_{\eta} \partial_{t}\left(\Lambda_{\alpha}-\Lambda_{0}\right)(\tilde{f})(x, t) d \sigma(x) d t=-\int_{0}^{T} \int_{\Gamma} \partial_{t}^{2} \theta_{\eta}\left(\Lambda_{\alpha}-\Lambda_{0}\right)(\tilde{f})(x, t) d \sigma(x) d t
$$

On the other hand, we have

$$
\begin{aligned}
& \int_{0}^{T} \int_{\Gamma}\left[\theta_{\eta}\left(\Lambda_{\alpha}-\Lambda_{0}\right)(\tilde{f})+\partial_{t} \theta_{\eta} \partial_{t}\left(\Lambda_{\alpha}-\Lambda_{0}\right)(\tilde{f})\right](x, t) d \sigma(x) d t \\
& =\int_{0}^{T} \int_{\Gamma}\left[\theta_{\eta}\left(\Lambda_{\alpha}(\tilde{f})-\tilde{\Lambda}_{\alpha}\left(\left.\tilde{u}_{\alpha}\right|_{\Gamma \times(0, T)}\right)\right)+\partial_{t} \theta_{\eta} \partial_{t}\left(\Lambda_{\alpha}(\tilde{f})-\tilde{\Lambda}_{\alpha}\left(\left.\tilde{u}_{\alpha}\right|_{\Gamma \times(0, T)}\right)\right)\right](x, t) d \sigma(x) d t \\
& \quad+\int_{0}^{T} \int_{\Gamma}\left[\theta_{\eta} \alpha^{d} \int_{0}^{t} e^{-i|\eta| s} \frac{\partial v_{\eta}}{\partial n}(x, t-s) d s+\alpha^{d} \partial_{t} \theta_{\eta} \partial_{t} \int_{0}^{t} e^{-i|\eta| s} \frac{\partial v_{\eta}}{\partial n}(x, t-s) d s\right] d \sigma(x) d t,
\end{aligned}
$$

where $\tilde{\Lambda}_{\alpha}\left(\left.\tilde{u}_{\alpha}\right|_{\Gamma \times(0, T)}\right)=\Lambda_{0}(\tilde{f})+\alpha^{d} \int_{0}^{t} e^{-i|\eta| s} \Lambda_{0}\left(\left.v_{\eta}\right|_{\Gamma}\right)(x, t-s) d s$. 
Given that, $\theta_{\eta}$ satisfies the Volterra equation (3.4) and

$$
\begin{aligned}
\partial_{t}\left(\int_{0}^{t} e^{-i|\eta| s} \frac{\partial v_{\eta}}{\partial n}(x, t-s) d s\right) & =\partial_{t}\left(-e^{-i|\eta| t} \int_{0}^{t} e^{i|\eta| s} \frac{\partial v_{\eta}}{\partial n}(x, s) d s\right) \\
& =i|\eta| e^{-i|\eta| t} \int_{0}^{t} e^{i|\eta| s} \frac{\partial v_{\eta}}{\partial n}(x, s) d s+\frac{\partial v_{\eta}}{\partial n}(x, t),
\end{aligned}
$$

we obtain by integrating by parts over $(0, T)$ that

$$
\begin{aligned}
& \int_{0}^{T} \int_{\Gamma}\left[\theta_{\eta} \int_{0}^{t} e^{-i|\eta| s} \frac{\partial v_{\eta}}{\partial n}(x, t-s) d s+\partial_{t} \theta_{\eta} \partial_{t} \int_{0}^{t} e^{-i|\eta| s} \frac{\partial v_{\eta}}{\partial n}(x, t-s) d s\right] d \sigma(x) d t \\
& =\int_{0}^{T} \int_{\Gamma}\left(\frac{\partial v_{\eta}}{\partial n}(x, t)\left(\partial_{t} \theta_{\eta}+\int_{t}^{T} \theta_{\eta}(s) e^{i|\eta|(t-s)} d s\right)\right. \\
& \left.-i|\eta|\left(e^{-i|\eta| t} \partial_{t} \theta_{\eta}(t)\right) \int_{0}^{t} e^{i|\eta| s} \frac{\partial v_{\eta}}{\partial \eta}(x, s) d s\right) d \sigma(x) d t \\
& =\int_{0}^{T} \int_{\Gamma} \frac{\partial v_{\eta}}{\partial n}(x, t)\left(\partial_{t} \theta_{\eta}+\int_{t}^{T}\left(\theta_{\eta}(s)-i|\eta| \partial_{t} \theta_{\eta}(s)\right) e^{i|\eta|(t-s)} d s\right) d \sigma(x) d t \\
& =\int_{0}^{T} \int_{\Gamma} g_{\eta}(x, t) \Lambda_{0}\left(v_{\eta} \mid \Gamma\right)(x, t) d \sigma(x) d t
\end{aligned}
$$

and so, from Proposition 3.1, we obtain

$$
\begin{aligned}
& \int_{0}^{T} \int_{\Gamma}\left[\theta_{\eta}\left(\Lambda_{\alpha}-\Lambda_{0}\right)(\tilde{f})+\partial_{t} \theta_{\eta} \partial_{t}\left(\Lambda_{\alpha}-\Lambda_{0}\right)(\tilde{f})\right](x, t) d \sigma(x) d t \\
& =\alpha^{d}|\eta|^{2} \int_{\Omega^{\prime}} c_{1}(x) e^{2 i \eta \cdot x} d x \\
& \quad+\int_{0}^{T} \int_{\Gamma}\left[\theta_{\eta}\left(\Lambda_{\alpha}(\tilde{f})-\tilde{\Lambda}_{\alpha}\left(\left.\tilde{u}_{\alpha}\right|_{\Gamma \times(0, T)}\right)\right)+\partial_{t} \theta_{\eta} \partial_{t}\left(\Lambda_{\alpha}(\tilde{f})-\tilde{\Lambda}_{\alpha}\left(\left.\tilde{u}_{\alpha}\right|_{\Gamma \times(0, T)}\right)\right)\right] d \sigma(x) d t \\
& \quad+O\left(\alpha^{d+1}\right) .
\end{aligned}
$$

Thus, to prove Theorem 3.3, it suffices then to show that

$$
\int_{0}^{T} \int_{\Gamma}\left[\theta_{\eta}\left(\Lambda_{\alpha}(\tilde{f})-\tilde{\Lambda}_{\alpha}\left(\left.\tilde{u}_{\alpha}\right|_{\Gamma \times(0, T)}\right)\right)+\partial_{t} \theta_{\eta} \partial_{t}\left(\Lambda_{\alpha}(\tilde{f})-\tilde{\Lambda}_{\alpha}\left(\left.\tilde{u}_{\alpha}\right|_{\Gamma \times(0, T)}\right)\right)\right] d \sigma(x) d t=O\left(\alpha^{d+1}\right) .
$$


Journal of Applied Mathematics

From definition (3.10), we have

$$
\widehat{u}_{\alpha}-\widehat{\tilde{u}}_{\alpha}=\int_{0}^{T}\left(u_{\alpha}-\tilde{u}_{\alpha}\right) z(t) d t
$$

which gives by system (3.24) that

$$
\begin{aligned}
& \Delta\left(\widehat{u}_{\alpha}-\widehat{\widetilde{u}}_{\alpha}\right) \\
& =\int_{0}^{T} c_{\alpha} \partial_{t}^{2}\left(u_{\alpha}-\tilde{u}_{\alpha}\right) z(t) d t+\alpha^{d} \int_{0}^{T} \nabla \cdot\left(c_{1}(x) \operatorname{grad}\left(\int_{0}^{t} e^{-i|\eta| s} v_{\eta}(x, t-s) d s\right)\right) z(t) d t .
\end{aligned}
$$

Thus, by (3.9) and (3.24) again, we see that the function $\widehat{u}_{\alpha}-\tilde{u}_{\alpha}$ is the solution of

$$
\begin{gathered}
-\Delta\left(\widehat{u}_{\alpha}-\widehat{\tilde{u}}_{\alpha}\right)=-\int_{0}^{T} c_{\alpha}\left(u_{\alpha}-\tilde{u}_{\alpha}\right) z^{\prime \prime}(t) d t+\nabla \cdot\left(c_{1}(x) \operatorname{grad}\left(\widehat{\tilde{u}}_{\alpha}-\widehat{u}\right)\right) \quad \text { in } \Omega \\
\left.\left(\widehat{u}_{\alpha}-\widehat{\tilde{u}}_{\alpha}\right)\right|_{\partial \Omega}=0 .
\end{gathered}
$$

Taking into account estimate (3.12) given by Lemma 3.2, then by using standard elliptic regularity (see, e.g., [24]) for the boundary value problem (3.38), we find that

$$
\left\|\frac{\partial}{\partial n}\left(\widehat{u}_{\alpha}-\widehat{\widetilde{u}}_{\alpha}\right)\right\|_{L^{2}(\Gamma)}=O\left(\alpha^{d+1}\right)
$$

By the fact that $\Lambda_{\alpha}(\tilde{f})-\tilde{\Lambda}_{\alpha}\left(\left.\tilde{u}_{\alpha}\right|_{\Gamma \times(0, T)}\right):=(\partial / \partial n)\left(u_{\alpha}-\tilde{u}_{\alpha}\right) \in L^{\infty}\left(0, T ; L^{2}(\Gamma)\right)$, we deduce, as done in the proof of Lemma 3.2, that

$$
\left\|\Lambda_{\alpha}(\tilde{f})-\tilde{\Lambda}_{\alpha}\left(\left.\tilde{u}_{\alpha}\right|_{\Gamma \times(0, T)}\right)\right\|_{L^{2}(\Gamma)}=O\left(\alpha^{d+1}\right)
$$

which implies that

$$
\int_{0}^{T} \int_{\Gamma}\left[\theta_{\eta}\left(\Lambda_{\alpha}(\tilde{f})-\tilde{\Lambda}_{\alpha}\left(\left.\tilde{u}_{\alpha}\right|_{\Gamma \times(0, T)}\right)\right)+\partial_{t} \theta_{\eta} \partial_{t}\left(\Lambda_{\alpha}(\tilde{f})-\tilde{\Lambda}_{\alpha}\left(\left.\tilde{u}_{\alpha}\right|_{\Gamma \times(0, T)}\right)\right)\right] d \sigma(x) d t=O\left(\alpha^{d+1}\right) .
$$

This completes the proof of our Theorem. 
We are now in position to describe our identification procedure which is based on Theorem 3.3. Let us neglect the asymptotically small remainder in the asymptotic formula (3.27). Then, it follows that

$$
c_{\alpha}(x)-c_{0}(x) \approx \frac{2}{\alpha^{d-1}} \int_{\mathbb{R}^{d}} \frac{e^{-2 i \eta \cdot x}}{|\eta|^{2}} \int_{0}^{T} \int_{\Gamma}\left(\theta_{\eta}+\partial_{t} \theta_{\eta} \partial_{t} \cdot\right)\left(\Lambda_{\alpha}-\Lambda_{0}\right)(\tilde{f})(x, t) d \sigma(y) d t d \eta, \quad x \in \Omega .
$$

The method of reconstruction we propose here consists in sampling values of

$$
\frac{1}{|\eta|^{2}} \int_{0}^{T} \int_{\Gamma}\left(\theta_{\eta}+\partial_{t} \theta_{\eta} \partial_{t}\right)\left(\Lambda_{\alpha}-\Lambda_{0}\right)(\tilde{f})(x, t) d \sigma(x) d t
$$

at some discrete set of points $\eta$ and then calculating the corresponding inverse Fourier transform.

In the following, a better approximation than (2.3) is derived. It is not hard to prove the more convenient approximation in terms of the values of local Dirichlet-to-Neumann maps $\Lambda_{\alpha}$ and $\Lambda_{0}$ at $\tilde{f}$.

Corollary 3.4. Let $\eta \in \mathbb{R}^{d}$ and let $\tilde{f}=\left.f\right|_{\Gamma} \in \widetilde{H}^{1 / 2}(\Gamma)$. Suppose that $\Gamma$ and $T$ geometrically control $\Omega$; then we have the following better approximation:

$$
\begin{aligned}
& c_{\alpha}(x) \approx c_{0}(x) \\
& \quad-\frac{2}{\alpha^{d-1}} \int_{\mathbb{R}^{d}} \frac{e^{-2 i \eta \cdot x}}{|\eta|^{2}} \int_{0}^{T} \int_{\Gamma}\left[e^{i|\eta| t} \partial_{t}\left(e^{-i|\eta| t} g_{\eta}(y, t)\right)\left(\Lambda_{\alpha}-\Lambda_{0}\right)(\tilde{f})(y, t)\right] d \sigma(y) d t d \eta, \quad x \in \Omega,
\end{aligned}
$$

where the boundary control $g_{\eta}$ is defined by (3.2).

Proof. The term $\int_{0}^{T} \int_{\Gamma} \partial_{t} \theta_{\eta} \partial_{t}\left(\Lambda_{\alpha}-\Lambda_{0}\right)(\tilde{f})(x, t) d \sigma(x) d t$, given in Theorem 3.3, has to be interpreted as follows:

$$
\int_{0}^{T} \int_{\Gamma} \partial_{t} \theta_{\eta} \cdot \partial_{t}\left(\Lambda_{\alpha}-\Lambda_{0}\right)(\tilde{f})(x, t) d \sigma(x) d t=-\int_{0}^{T} \int_{\Gamma} \partial_{t}^{2} \theta_{\eta} \cdot\left(\Lambda_{\alpha}-\Lambda_{0}\right)(\tilde{f})(x, t) d \sigma(x) d t
$$

because $\left.\theta_{\eta}\right|_{t=T}=0$ and $\left.\partial_{t}\left(\partial u_{\alpha} / \partial n-\partial u / \partial n\right)\right|_{t=0}=0$. In fact, in view of the ODE (3.4), the term $\int_{0}^{T} \int_{\Gamma}\left[\theta_{\eta}\left(\Lambda_{\alpha}-\Lambda_{0}\right)+\partial_{t} \theta_{\eta} \cdot \partial_{t}\left(\Lambda_{\alpha}-\Lambda_{0}\right)\right] \tilde{f}(x, t) d \sigma(x) d t$ may be simplified after integration by parts over $(0, T)$ and using of the fact that $\theta_{\eta}$ is the solution to the ODE (3.4) to become

$$
-\int_{0}^{T} \int_{\Gamma} e^{i|\eta| t} \partial_{t}\left(e^{-i|\eta| t} g_{\eta}\right) \cdot\left(\Lambda_{\alpha}-\Lambda_{0}\right)(\tilde{f})(x, t) d \sigma(x) d t .
$$

Then, the desired approximation is established. 


\section{Conclusion}

The use of approximate formula (3.27), including the difference between the local Dirichlet to Neumann maps, represents a promising approach to the dynamical identification and reconstruction of a coefficient which is unknown in a bounded domain (but it is known outside of this domain) for a class of hyperbolic PDE. We believe that this method will yield a suitable approximation to the dynamical identification of small conductivity ball (of the form $z+\alpha D$ ) in a homogeneous medium in $\mathbb{R}^{d}$ from the boundary measurements. We will present convenable numerical implementations for this investigation. This issue will be considered in a forthcoming work.

\section{Acknowledgments}

The authors are grateful to the editor and the anonymous referees for their valuable comments and helpful suggestions which have much improved the presentation of the article.

\section{References}

[1] H. Ammari, "Identification of small amplitude perturbations in the electromagnetic parameters from partial dynamic boundary measurements," Journal of Mathematical Analysis and Applications, vol. 282, no. 2, pp. 479-494, 2003.

[2] L. Beilina and M. V. Klibanov, "A globally convergent numerical method for a coefficient inverse problem," SIAM Journal on Scientific Computing, vol. 31, no. 1, pp. 478-509, 2008.

[3] M. I. Belishev, "Dynamical inverse problem for the equation $-\Delta u-\nabla \ln \rho \cdot \nabla u=0$ (the BC method)," Cubo, vol. 10, no. 2, pp. 15-30, 2008.

[4] M. I. Belishev, "The Caldéron problem for two-dimensional manifolds by the BC-method," SIAM Journal on Mathematical Analysis, vol. 35, no. 1, pp. 172-182, 2003.

[5] S. I. Kabanikhin, Projection-Difference Methods for Determining the Coeffcients of Hyperbolic Equations, Nauka, Novosibirsk, Russia, 1988.

[6] M. V. Klibanov, "Inverse problems and Carleman estimates," Inverse Problems, vol. 8, no. 4, pp. 575596, 1992.

[7] A. I. Nachman, "Global uniqueness for a two-dimensional inverse boundary value problem," Annals of Mathematics, vol. 143, no. 1, pp. 71-96, 1996.

[8] A. G. Ramm, Inverse Problems: Mathematical and Analytical Techniques with Applications to Engineering, Springer, New York, NY, USA, 2005.

[9] A. G. Ramm and Rakesh, "Property $C$ and an inverse problem for a hyperbolic equation," Journal of Mathematical Analysis and Applications, vol. 156, no. 1, pp. 209-219, 1991.

[10] M. Yamamoto, "Stability, reconstruction formula and regularization for an inverse source hyperbolic problem by a control method," Inverse Problems, vol. 11, no. 2, pp. 481-496, 1995.

[11] M. Yamamoto, "Stability, reconstruction formula and regularization for an inverse source hyperbolic problem by a control method," Inverse Problems, vol. 11, no. 2, pp. 481-496, 1995.

[12] L. Beilina and M. V. Klibanov, "A globally convergent numerical method and the adaptivity technique for a hyperbolic coefficient inverse problem. Part I: analytical study," preprint, 2009.

[13] M. V. Klibanov and A. Timonov, "Numerical studies on the globally convergent convexification algorithm in 2D," Inverse Problems, vol. 23, no. 1, pp. 123-138, 2007.

[14] M. V. Klibanov and A. Timonov, Carleman Estimates for Coefficient Inverse Problems and Numerical Applications, Inverse and Ill-posed Problems Series, VSP, Utrecht, The Netherlands, 2004.

[15] J. Xin and M. V. Klibanov, "Comparative studies of the globally convergent convexification algorithm with application to imaging of antipersonnel land mines," Applicable Analysis, vol. 86, no. 9, pp. 11471176, 2007.

[16] L. Päivärinta and V. Serov, "Recovery of jumps and singularities in the multidimensional Schrödinger operator from limited data," Inverse Problems and Imaging, vol. 1, no. 3, pp. 525-535, 2007. 
[17] V. Serov and L. Päivärinta, "Inverse scattering problem for two-dimensional Schrödinger operator," Journal of Inverse and Ill-Posed Problems, vol. 14, no. 3, pp. 295-305, 2006.

[18] Yu. Chen, "Inverse scattering via Heisenberg's uncertainty principle," Inverse Problems, vol. 13, no. 2, pp. 253-282, 1997.

[19] C. Daveau, A. Khelifi, and A. Sushchenko, "Reconstruction of closely spaced small inhomogeneities via boundary measurements for the full time-dependent Maxwell's equations," Applied Mathematical Modelling, vol. 33, no. 3, pp. 1719-1728, 2009.

[20] D. J. Cedio-Fengya, S. Moskow, and M. S. Vogelius, "Identification of conductivity imperfections of small diameter by boundary measurements. Continuous dependence and computational reconstruction," Inverse Problems, vol. 14, no. 3, pp. 553-595, 1998.

[21] A. Friedman and M. Vogelius, "Identification of small inhomogeneities of extreme conductivity by boundary measurements: a theorem on continuous dependence," Archive for Rational Mechanics and Analysis, vol. 105, no. 4, pp. 299-326, 1989.

[22] J. Sylvester and G. Uhlmann, "A global uniqueness theorem for an inverse boundary value problem," Annals of Mathematics, vol. 125, no. 1, pp. 153-169, 1987.

[23] H. Brezis, Analyse Fonctionnelle, Collection Mathématiques Appliquées pour la Maîtrise, Masson, Paris, France, 1983.

[24] L. C. Evans, Partial Differential Equations, vol. 19 of Graduate Studies in Mathematics, American Mathematical Society, Providence, RI, USA, 1998.

[25] J.-L. Lions, Contrôlabilité Exacte, Perturbations et Stabilisation de Systèmes Distribués. Tome 1, Contrôlabilité Exacte, vol. 8 of Recherches en Mathématiques Appliquées, Masson, Paris, France, 1988.

[26] M. I. Belishev and Y. V. Kurylev, "Boundary control, wave field continuation and inverse problems for the wave equation," Computers \& Mathematics with Applications, vol. 22, no. 4-5, pp. 27-52, 1991.

[27] G. Bruckner and M. Yamamoto, "Determination of point wave sources by pointwise observations: stability and reconstruction," Inverse Problems, vol. 16, no. 3, pp. 723-748, 2000.

[28] J.-P. Puel and M. Yamamoto, "Applications de la contrôlabilité exacte à quelques problèmes inverses hyperboliques," Comptes Rendus de l'Académie des Sciences. Série I. Mathématique, vol. 320, no. 10, pp. 1171-1176, 1995.

[29] J.-P. Puel and M. Yamamoto, "On a global estimate in a linear inverse hyperbolic problem," Inverse Problems, vol. 12, no. 6, pp. 995-1002, 1996.

[30] Rakesh and W. W. Symes, "Uniqueness for an inverse problem for the wave equation," Communications in Partial Differential Equations, vol. 13, no. 1, pp. 87-96, 1988.

[31] Z. Q. Sun, "On continuous dependence for an inverse initial-boundary value problem for the wave equation," Journal of Mathematical Analysis and Applications, vol. 150, no. 1, pp. 188-204, 1990.

[32] C. Bardos, G. Lebeau, and J. Rauch, "Sharp sufficient conditions for the observation, control, and stabilization of waves from the boundary," SIAM Journal on Control and Optimization, vol. 30, no. 5, pp. 1024-1065, 1992.

[33] M. Yamamoto, "On an inverse problem of determining source terms in Maxwell's equations with a single measurement," in Inverse Problems, Tomography, and Image Processing (Newark, DE, 1997), pp. 241-256, Plenum, New York, NY, USA, 1998.

[34] N. U. Ahmed and T. Wan, "Exact boundary controllability of electromagnetic fields in general regions," Dynamic Systems and Applications, vol. 5, no. 2, pp. 229-243, 1996. 


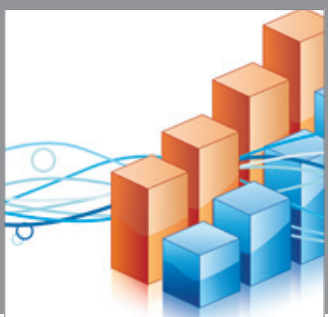

Advances in

Operations Research

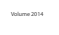

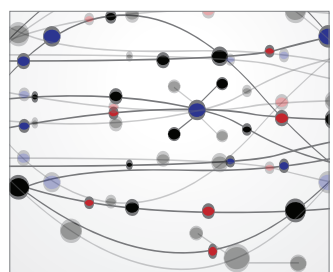

\section{The Scientific} World Journal
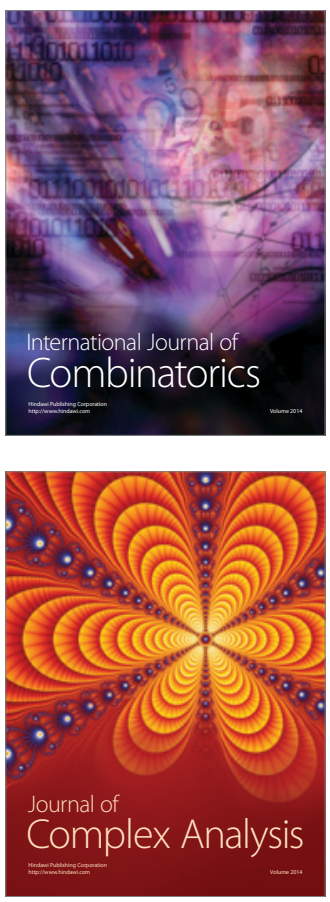

International Journal of

Mathematics and

Mathematical

Sciences
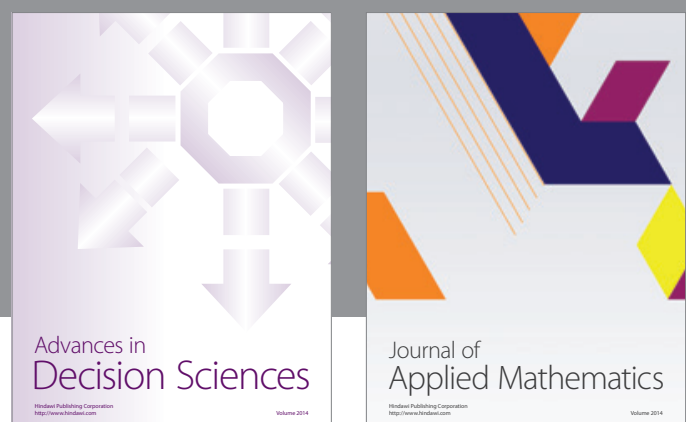

Journal of

Applied Mathematics
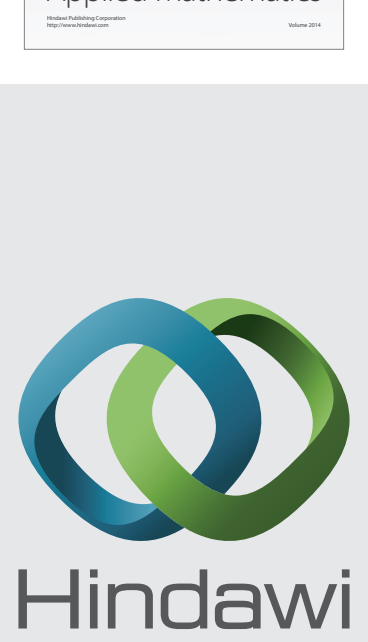

Submit your manuscripts at http://www.hindawi.com
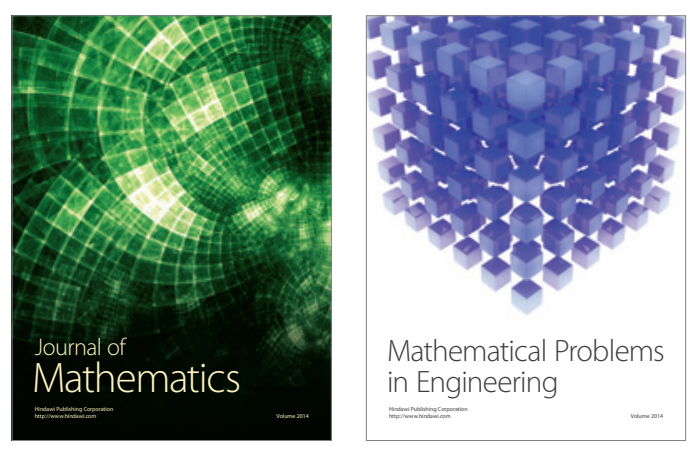

Mathematical Problems in Engineering
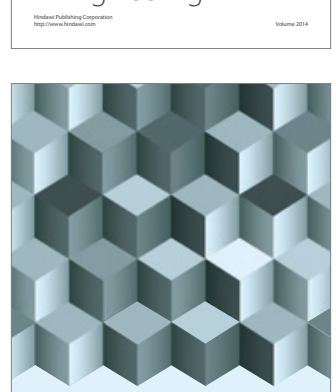

Journal of

Function Spaces
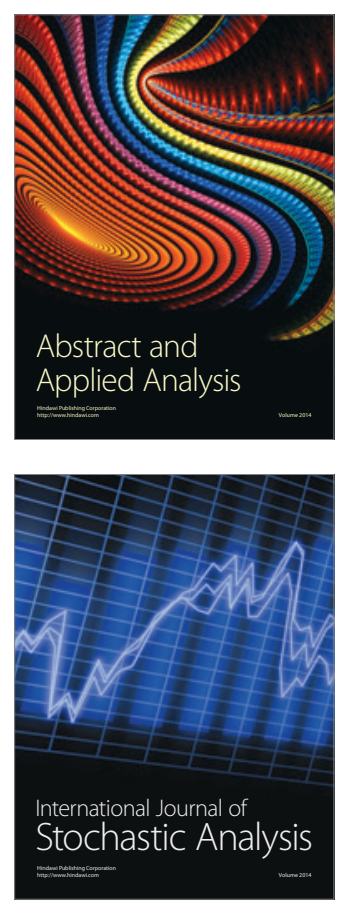

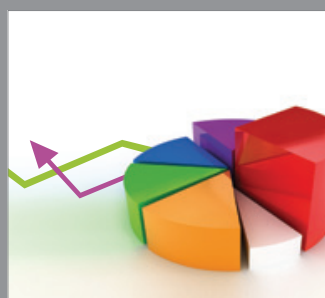

ournal of

Probability and Statistics

Promensencen
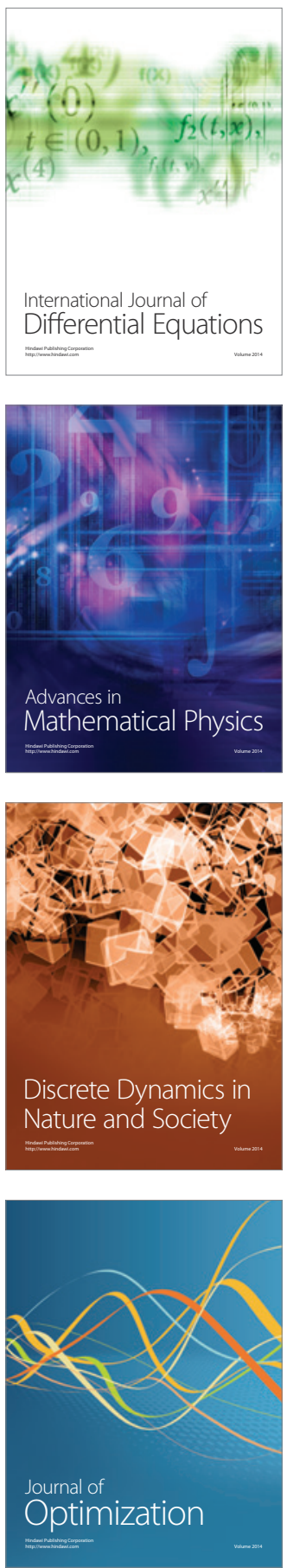